\title{
WAVELETS AND RADIAL BASIS FUNCTIONS: A UNIFYING PERSPECTIVE
}

\author{
Michael Unser and Thierry Blu \\ Biomedical Imaging Group \\ Swiss Federal Institute of Technology Lausanne \\ CH-1015 Lausanne EPFL, Switzerland \\ http://bigwww.epfl.ch/
}

\begin{abstract}
Wavelets and radial basis functions (RBF) are two rather distinct ways of representing signals in terms of shifted basis functions. An essential aspect of RBF, which makes the method applicable to non-uniform grids, is that the basis functions, unlike wavelets, are non-local-in addition, they do not involve any scaling at all. Despite these fundamental differences, we show that the two types of representation are closely connected. We use the linear splines as motivating example. These can be constructed by using translates of the one-side ramp function (which is not localized), or, more conventionally, by using the shifts of a linear B-spline. This latter function, which is the prototypical example of a scaling function, can be obtained by localizing the one-side ramp function using finite differences. We then generalize the concept and identify the whole class of self-similar radial basis functions that can be localized to yield conventional multiresolution wavelet bases. Conversely, we prove that, for any compactly supported scaling function $\varphi(x)$, there exists a one-sided central basis function $\rho_{+}(x)$ that spans the same multiresolution subspaces. The central property is that the multiresolution bases are generated by simple translation of $\rho_{+}$, without any dilation.
\end{abstract}

Keywords: Wavelets, radial basis functions, fractals, self-similarity, non-uniform grids, splines

\section{INTRODUCTION}

Radial basis functions constitute a powerful framework for interpolating or approximating data on non-uniform grids. ${ }^{6}$ Given a set of multidimensional grid points $\mathbf{x}_{k} \in \mathbb{R}^{p}$ and a suitable radial function $\rho(r): \mathbb{R}_{+} \rightarrow \mathbb{R}$, the generic form of the representation is

$$
\forall \mathbf{x} \in \mathbb{R}^{p}, f(\mathbf{x})=\sum_{k} a_{k} \rho\left(\left\|\mathbf{x}-\mathbf{x}_{k}\right\|\right)
$$

where $\|\cdot\|$ denotes the Euclidean distance. The basis functions in (1) depend only on the distance to their corresponding grid point $\mathbf{x}_{k}$ and are thus called radial. The $a_{k}$ 's are weighting coefficients that are typically determined by fitting the function to some data points $y_{k}=f\left(\mathbf{x}_{k}\right)$ (solution of a linear system of equations). Often, the formulation also includes a regularization term which specifies the optimal radial basis function $\rho(r)$ implicitely. ${ }^{4}$

Formula (1) is reminiscent of a wavelet-like expansion in terms of translates of a scaling function $\varphi(x)$. However, there are fundamental differences that need to be emphazised. First, (1) has no provision for scaling; the basis functions remain the same irrespective of the distance between the data points. Since the RBF approach must be valid for arbitrary grids, this clearly excludes the use of basis functions that are compactly supported. Second, the basis functions in both representations are fundamentally different. With wavelets, the scaling functions $\varphi(x)$ are well localized (typ., compactly supported). Radial basis functions, by contrast, are typically increasing and unbounded at infinity and therefore not even square integrable. Third, the radial basis function framework is ideally suited to a non-uniform multivariate setting while conventional wavelet theory is restricted to uniform grids, preferably in 1D. Thus, radial basis functions are more general than wavelets, but also more difficult to handle because of poor conditioning.

Email addresses: Michael.Unser@epfl.ch, Thierry.Blu.epfl.ch 
It is well known in approximation theory that both formalisms are applicable to the construction of polynomial splines. The so-called wavelet approach uses the B-splines as basis functions; it can be traced back to the pioneering work of Schoenberg. ${ }^{7}$ The alternative representation uses non-local basis functions which are either one-sided or radial: $x_{+}^{n}$ or $|x|^{n}$, assuming that the degree $n$ of the spline is even. For $p=1$, the two methods are rigorously equivalent. This equivalence and its relevance for multiresolution approximation will be explained in more details in Section 2 using the linear splines as motivating example.

In this paper, we will show that the equivalence that holds between radial basis functions and splines can also be established for wavelets in general. For this purpose, we will restrict ourselves to the standard univariate wavelet setting where the grid is uniform. We will also introduce the notion of central basis function to break the symmetry of the radial ones. We will approach the problem from its two opposite sides. First, in Section 3, we will show how to construct scaling functions (or wavelets) starting from some central basis function, extending earlier results of Buhmann and Utreras. ${ }^{3,9}$ In particular, we will identify a necessary self-similarity condition for $\rho(r)$ and derive a complete characterization of the relevant class of central functions. In Section 4, we will consider the converse implication and prove that any standard multiresolution analyis of $L_{2}$ can be expressed in terms of central basis functions. In other words, we will uncover the radial basis function that lies hidden within any scaling function or wavelet. We will also present examples to illustrate our results.

\section{MOTIVATION: THE EXAMPLE OF LINEAR SPLINES}

The best way to motivate our investigation is to start with a concrete example. We will thus build a multiresolution of $L_{2}$ using piecewise linear functions but we will proceed in a non-standard fashion.

\subsection{Splines and one-sided power functions}

function, The basic space of piecewise linear splines with knots at the integers can be specified in terms of the integer

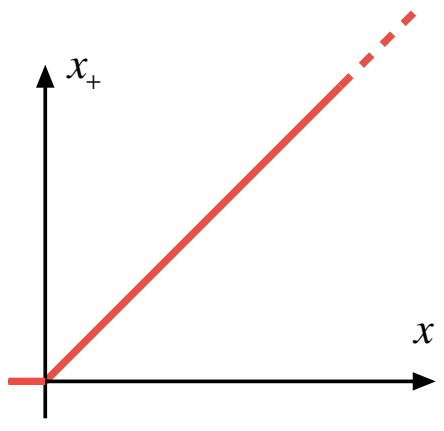

(a)

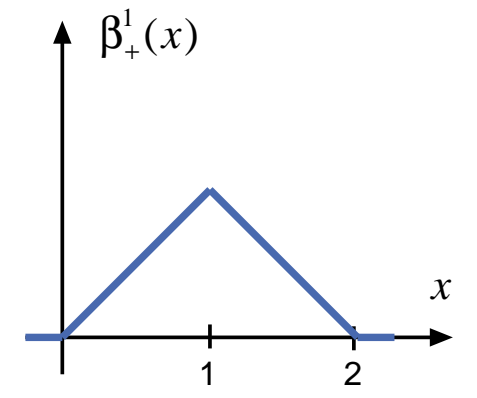

(b)

Figure 1. The example of linear splines: (a) one-sided ramp function, (b) B-spline of degree 1. The function in (b) is localized, while the one in (a) is not.

shifts of the one-sided linear ramp function (cf. Fig. 1a):

$$
V_{0}=\operatorname{span}\left\{\rho_{+}(x-k)\right\}_{k \in \mathbb{Z}}
$$

with $\rho_{+}(x)=x_{+}=\max \{x, 0\}$. Since $x_{+}$has a single singularity at the origin, each of the basis functions is clearly associated with one of the spline knots. The ramp function is thus well adapted to the mathematical structure of linear splines. However, it has the disadvantage of not being local and is therefore rarely used for performing numerical computations. 1)

By taking the 2nd forward finite difference of $\rho_{+}(x)$, one generates the hat function (or causal B-spline of degree

$$
\beta_{+}^{1}(x)=\Delta_{+}^{2} x_{+}=x_{+}-2(x-1)_{+}+(x-2)_{+}
$$


This function, which is shown in Fig. 1b, is the more standard, compactly supported basis function for the linear splines. Interestingly, we can also invert (3) and express the one-sided power function as a weighted sum of B-spline basis functions

$$
x_{+}=\sum_{k \geq 0}(k+1) \beta_{+}^{1}(x-k)
$$

This shows that our definition (2) of the basic spline space is equivalent to the standard one which involves linear combinations of B-splines. Thus $\left\{\rho_{+}(x-k)\right\}_{k \in \mathbb{Z}}$ is a valid basis for $V_{0}$, albeit not a Riesz basis, since the functions are not square-integrable.

\subsection{How multiresolution becomes trivial}

The present formulation makes the multiresolution structure of splines stand out quite naturally (cf. Fig. 2). Consider the fine-to-coarse sequence of subspaces $\cdots V_{0} \supset V_{1} \cdots \supset V_{i} \cdots$, where $V_{i}$ represents the space of linear splines with knots at $x_{k}=2^{i} k, k \in \mathbb{Z}$. These splines are generated simply by dropping all the basis functions in (2) that are not positioned at the desired knots. Thus, we define our uniform spline space are scale $a=2^{i}$ as

$$
V_{i}=\operatorname{span}\left\{\rho_{+}\left(x-2^{i} k\right)\right\}_{k \in \mathbb{Z}}
$$

It is important to note that these multiresolution basis functions are generated by translation only - no dilation is required. Clearly, the basis functions for $V_{i}$ are a subset of those of $V_{j}$ for $j<i$, which implies that $V_{j} \supset V_{i}$, for all $j<i$ (multiresolution property). Since each $V_{i}$ also has a B-spline Riesz basis $\left\{2^{-i / 2} \beta_{+}^{1}\left(x / 2^{i}-k\right)\right\}_{k \in \mathbb{Z}}$, the whole ladder of spline subspaces for $i \in \mathbb{Z}$ generates a multiresolution of $L_{2}$ as defined by Mallat. ${ }^{5}$ Hence, it is possible to construct a whole variety of corresponding wavelet bases using any of the standard design techniques.
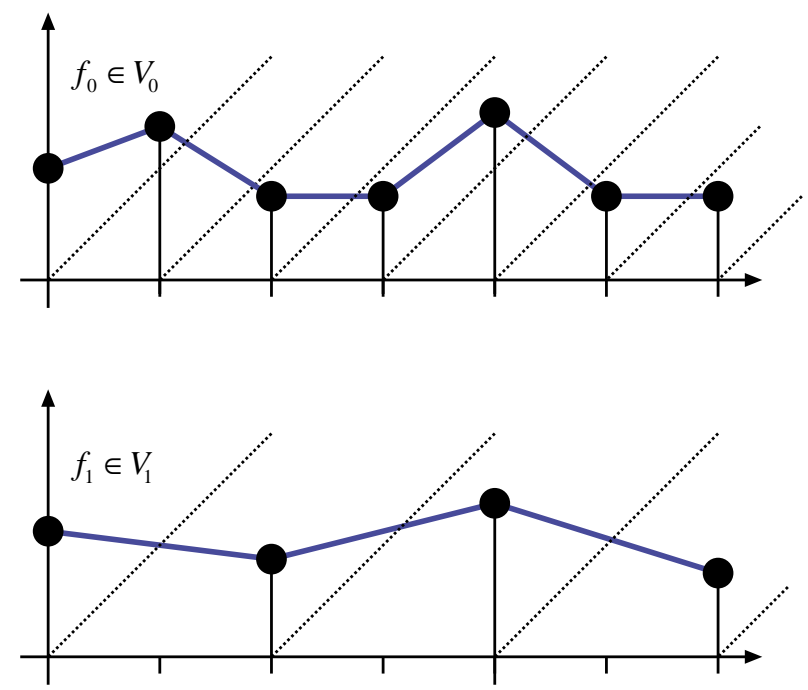

Figure 2. Multiresolution spaces using one-sided power functions.

\subsection{Non-uniform linear splines}

The power of the present formulation really becomes apparent if we move one step further and consider a given non-uniform sequence of knots $\cdots<x_{k}<x_{k+1}<\cdots$ with $k \in \mathbb{Z}$. We then define a corresponding embedded sequence of non-uniform spline spaces

$$
V_{i}=\operatorname{span}\left\{\rho_{+}\left(x-x_{2^{i} k}\right)\right\}_{k \in \mathbb{Z}}
$$

which share the same inclusion properties as before: $V_{0} \supset V_{1} \cdots \supset V_{i} \cdots$. Here too, we are able to produce compactly supported basis functions (non-uniform B-splines), except that they lose the convenient shift-invariant structure that is inherent to standard (uniform) multiresolution analysis. They are the triangular functions that take the value one at $x=x_{2^{i}(k+1)}$ and vanish for $x \leq x_{2^{i} k}$ and $x \geq x_{2^{i}(k+2)}$. These can be constructed using the 2 nd divided differences of $\rho_{+}(x)$ rather than finite differences as in (3). This non-uniform setting is also suitable for constructing wavelet bases which span the orthogonal complement of $V_{i}$ with respect to $V_{i-1} .^{2}$ 


\subsection{Central basis functions}

Until now, we have worked with the one-sided function $\rho_{+}(x)=x_{+}$. Another option would have been to use the symmetric function $\rho_{*}(x)=|x|$; the prototypical example of a radial basis function. In the sequel, we will consistently use the subscripts $*$, and + , to denote symmetric (or radial), and causal (or one-sided) generating functions, respectively. These are all special cases of what we call central basis functions and which we denote by the generic symbol $\rho(x)$. The one-sided functions are typically simpler to work with in the univariate case. The symmetric ones, on the other hand, are usually preferred in a multivariate setting, for they fall within the framework of the well-developed theory of radial basis functions. ${ }^{4,6}$

\section{FROM RADIAL BASIS FUNCTIONS TO WAVELETS}

We will now extend what we have just done with the ramp function and show how it is possible to construct wavelets starting from a radial (or central) basis function $\rho(x)$. We will not consider wavelets literally, but rather their associated scaling functions which are the key to the multiresolution structure of the wavelet transform. Once the scaling function has been specified, it is easy to construct a corresponding wavelet basis using standard techniques. ${ }^{5}$

\subsection{Scaling functions}

Often, a scaling function is defined indirectly through its refinement filter $h$ (cf. (8) below). One then has to worry about the delicate issues of the convergence of the iterated filterbank and of the $L_{2}$-completeness of the wavelet expansion. Here, we propose a more explicit definition that avoids these problems at the onset.

DEFINITION 3.1. $\varphi(x)$ is a valid scaling function if and only if it satifies the following three conditions:

(a) Riesz basis condition

$\{\varphi(x-k)\}_{k \in \mathbb{Z}}$ is a Riesz basis of $V_{0}$ if and only if there exists two positive constants $A$ and $B$ such that

$$
A \leq \sum_{k \in \mathbb{Z}}|\hat{\varphi}(\omega+2 \pi k)|^{2} \leq B
$$

almost everywhere, where $\hat{\varphi}(\omega)$ is the Fourier transform of $\varphi(x)$.

(b) Two-scale relation

$$
\varphi(x / 2)=\sum_{k \in \mathbb{Z}} h(k) \varphi(x-k)
$$

(c) Partition of unity

$$
\sum_{k \in \mathbb{Z}} \varphi(x-k)=1 \Leftrightarrow \hat{\varphi}(2 \pi k)=\delta_{k}
$$

We can show that these three conditions are necessary and sufficient for $\varphi$ to generate a multiresolution analysis of $L_{2}$ in the sense defined by Mallat. ${ }^{5}$

\subsection{Admissible central basis functions}

What distinguishes radial basis functions from scaling functions is that they are not compactly supported and typically unbounded at infinity; this means that they are usually not even in $L_{2}$.

We will say that a central basis function is admissible if it can reproduce the constant (first order of approximation) and if it can be localized in $L_{2}$ through a suitable choice of linear combinations (similar to taking the 2nd finite difference of the one-sided ramp function). The first condition is satisfied if the Fourier transform of $\rho(x)$ has at least a singularity of order 1 at the origin: $\hat{\rho}(\omega)=O\left(\omega^{-\gamma}\right)$ with $\gamma \geq 1$ as $\omega \rightarrow 0$. (cf. the work of Buhmann ${ }^{1}$ ). The second condition means that there must exist a sequence $p_{k}$ such that the function

$$
\varphi(x)=\sum_{k \in \mathbb{Z}} p_{k} \rho(x-k)
$$

satisfies the Riesz basis condition (7). 
One localization approach is the orthogonalization of $\rho(x)$, which is best described in the Fourier domain:

$$
\hat{\phi}(\omega)=\frac{\hat{\rho}(\omega)}{\sqrt{\sum_{n \in \mathbb{Z}}|\hat{\rho}(\omega+2 \pi n)|^{2}}}
$$

This technique is always applicable when the function is admissible, but it is not necessarily the simplest nor the most efficient one.

\subsection{Self-similar central basis functions}

The last ingredient that is missing to construct scaling functions is the two-scale relation (8). We can now state our first theoretical results.

THEOREM 3.2. An admissible central basis function $\rho(x)$ generates a multiresolution of $L_{2}$ if

$$
\rho(x)=\lambda \rho\left(\frac{x}{2}\right) .
$$

Thus, the key property for constructing wavelets is that the central basis functions be self-similar and therefore fractal.

A complete characterization of these functions is given by the following expansion

$$
\rho(x)=\sum_{n \in \mathbb{Z}} \gamma_{n} x_{+}^{\frac{\log \lambda}{\log 2}+j \frac{2 n \pi}{\log 2}}
$$

where the $\gamma_{n}$ can be viewed as free parameters, and where $x_{+}$is the one-sided ramp function already encountered previously. To obtain this formula, we consider the function $\rho_{0}(x)=x^{-\frac{\log \lambda}{\log 2}} \rho(x)$, and observe that $\rho_{0}\left(2^{x}\right)$ is $1-$ periodic. Thus, we may represent $\rho_{0}\left(2^{x}\right)$ in the distributional sense by its Fourier series $\rho_{0}\left(2^{x}\right)=\sum_{n \in \mathbb{Z}} c_{n} e^{j 2 n \pi x}$ which is equivalent to (13).

Conversely, we can obtain the coefficients $\gamma_{n}$ in (13) if we know the function $\rho(x)$ :

$$
\gamma_{n}=\frac{1}{\log 2} \int_{1}^{2} \rho(\xi) \xi^{-\frac{\log \lambda}{\log 2}-1-j \frac{2 n \pi}{\log 2}} d \xi
$$

This last expression simply follows from the standard formula for the Fourier coefficients of the function $\rho_{0}\left(2^{x}\right)$.

Formula (13) is very general but it also has its mathematical difficulties: it does not guarantee admissibility and its Fourier transform is only defined in the sense of distributions. It should therefore be handled with great precaution.

\subsection{Example of fractional splines}

If we limit ourselves to the first term of formula (13), we get a rather interesting family of functions: the fractional splines. $^{8}$ The corresponding basis functions are the one-sided power functions

$$
\rho_{+, \lambda}(x)=x_{+}^{\frac{\log \lambda}{\log 2}}
$$

which generate the fractional splines of degree $\alpha=\frac{\log \lambda}{\log 2}$. In particular, for $\lambda=2$, we are back to our introductory example: the piecewise linear splines. More generally, the one-side power functions $x_{+}^{\alpha}$ can be localized using fractional finite differences to yield the fractional B-splines ${ }^{8}$

$$
\beta_{+}^{\alpha}(x)=\frac{\Delta_{+}^{\alpha+1} x_{+}^{\alpha}}{\Gamma(\alpha+1)}
$$

Here, $\Delta_{+}^{\alpha+1} \longleftrightarrow\left(1-e^{-j \omega}\right)^{\alpha+1}$ is the causal fractional difference operator, and $\Gamma(\alpha+1)$ is Euler's gamma function, which generalizes the factorials. For $\alpha>-1 / 2$, the fractional B-splines are perfectly valid scaling functions in the sense of Definition 1. Presently, the fractional splines are the only known wavelet family with a continuously-varying order parameter $\alpha$. 


\section{FROM WAVELETS TO CENTRAL BASIS FUNCTIONS}

We now show that one can also follow the reverse path and uncover the central basis function(s) that lies hidden within any scaling function $\varphi(x)$.

THEOREM 4.1. Let $\varphi(x)$ be a compactly supported scaling function with corresponding refinement filter $H(z)=$ $\sum_{k=0}^{N} h_{k} z^{-k}$ with $h_{0} \neq 0$. Then, there exists a (non-unique) one-sided central basis function $\rho_{+}(x)$ that generates the same multiresolution analysis. One possible solution is

$$
\rho_{+}(x)=\sum_{n \geq 0} \rho_{n} \varphi(x-n)
$$

where the $\rho_{n}$ 's are generated as follows

$$
\begin{aligned}
& \rho_{0}=1 \\
& \rho_{n}=\frac{1}{h_{0}} \sum_{k} h_{n-2 k} \rho_{k}, \quad \forall n>0 .
\end{aligned}
$$

It is easy to verify that $\rho_{+}(x)$ is supported in $[0,+\infty)$ and that $\rho_{+}(x)=h_{0}^{-1} \rho_{+}(x / 2)$.

For the linear splines, we have $H(z)=\left(z+2+z^{-1}\right) / 2$, and the application of the theorem directly yields (4). We can also apply this result to the Daubechies wavelets which are orthogonal in addition to being compactly supported. The scaling and one-sided basis functions for the Daubechies wavelet D2 are shown in Fig. 3.

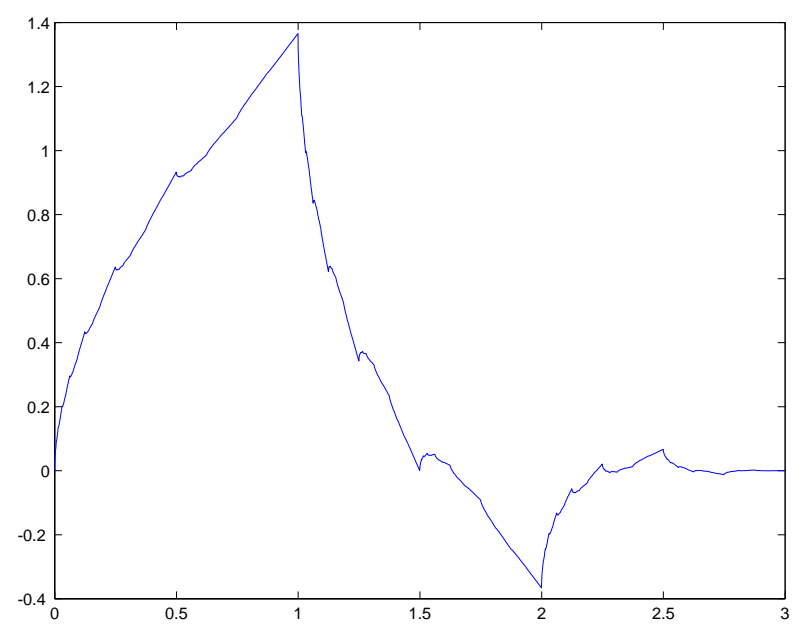

(a)

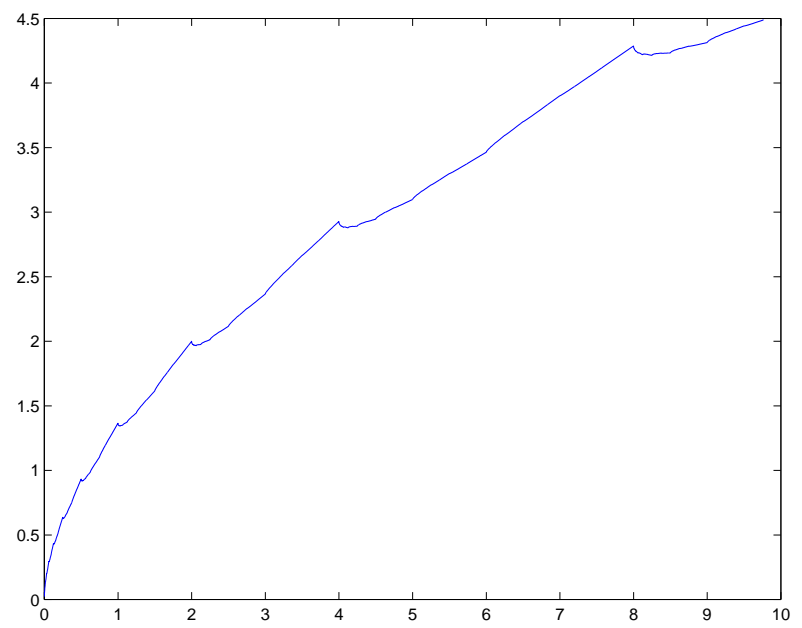

(b)

Figure 3. The example of Daubechies D2: (a) scaling function $\varphi(x)$, (b) one sided function $\rho_{+}(x)$.

When the scaling functions are symmetric, there is a similar connection with radial basis functions.

COROLLARY 4.1. Let $\varphi(x)$ be a symmetric compactly supported scaling function with corresponding refinement filter $H(z)$. Then there exists a radial basis function that generates the same multiresolution analysis: $\rho_{*}(x)=\rho_{+}(|x|)$ where $\rho_{+}(x)$ is given by $(16)$.

\section{CONCLUSION}

We have presented new results that make the connection between radial basis functions and wavelets very explicit.

The fact that we can move from radial basis functions to wavelets enables us to controle some of their key mathematical properties: order of approximation and regularity. It may also yield wavelets that have an explicit analytical form, the fractional splines being a notable example.

The existence of a link in the reverse direction - from wavelets to central basis functions - is especially interesting conceptually. It leads to an alternative interpretation of multiresolution: basis functions are simply removed (resp. added) instead of being dilated (resp. contracted) as is usually the case. This opens up the door to many possible extensions, such as wavelets on non-uniform grids. 


\section{REFERENCES}

1. M.-D. Buhmann, "Multivariate cardinal interpolation with radial-basis functions," Constructive Approximation, vol. 6 , no. 3, pp. 225-255, 1990.

2. M.-D. Buhmann and C.A. Micchelli, "Spline prewavelets for non-uniform knots," Numer. Math., vol. 61, pp. 455-474, 1992.

3. M.-D. Buhmann, "Discrete least squares approximation and prewavelets from radial function spaces," Math. Proc. Cambridge Phil. Soc., vol. 114, no. 3, pp. 533-558, 1993.

4. J. Duchon, "Splines minimizing rotation-invariant semi-norms in Sobolev spaces," in Constructive Theory of Functions of Several Variables, W. Schempp and K. Zeller, Eds., Berlin: Springer-Verlag, pp. 85-100, 1977.

5. S.G. Mallat, "A theory of multiresolution signal decomposition: the wavelet representation," IEEE Trans. Pattern Anal. Machine Intell., vol. PAMI-11, no. 7, pp. 674-693, 1989.

6. M.J.D. Powell, "The theory of radial basis function approximation in 1990," in Advances in Numerical Analysis II: Wavelets, Subdivision Algorithms and Radial Functions, W.A. Light, Ed., Oxford Univ. Press, pp. 105-210, 1992.

7. I.J. Schoenberg, "Contribution to the problem of approximation of equidistant data by analytic functions," Quart. Appl. Math., vol. 4, pp. 45-99, 112-141, 1946.

8. M. Unser and T. Blu, "Fractional splines and wavelets," SIAM Review, vol. 42, no. 1, pp. 43-67, March 2000.

9. F.I. Utreras, "Multiresolution and pre-wavelets using radial basis functions," in Multivariate Approximation: From CAGD to Wavelets, K. Jetter and F.I. Utreras, Eds., Singapore: World Scientific, pp. 321-333, 1993. 